\title{
The Going to Stay at Home program: combining dementia caregiver training and residential respite care
}

\author{
Meredith Gresham, ${ }^{1}$ Megan Heffernan² and Henry Brodaty ${ }^{3}$ \\ ${ }^{1}$ The Dementia Centre, Hammond Care, Sydney, New South Wales, Australia \\ ${ }^{2}$ Dementia Collaborative Research Centre, University of New South Wales, Sydney, New South Wales, Australia \\ ${ }^{3}$ Dementia Collaborative Research Centre and Centre for Healthy Brain Ageing, University of New South Wales, Sydney, New South Wales, Australia
}

ABSTRACT

Background: Caring for persons with dementia is stressful for family caregivers. Caregiver training programs and respite care can reduce this stress and help maintain persons with dementia living longer in the community. We evaluated a program that combines caregiver training with a residential respite stay.

Methods: In total, 90 dyads of persons with dementia and their caregivers, in groups of 3-6 dyads, volunteered to participate in a five-day residential training program and were followed-up 6 and 12 months later. The primary outcome was caregiver depression; secondary outcomes were measures of caregiver burden, unmet needs, person with dementia behavioral symptoms, and the quality of life and function.

Results: Caregiver depression and burden were unchanged, despite decreasing function in persons with dementia. Caregivers' unmet needs and behavioral symptoms in persons with dementia decreased significantly. Compared to a group of persons with dementia admitted for routine residential respite care, there was a marked reduction in permanent placement over 12 months.

Conclusions: The Going to Stay at Home Program is a feasible and practicable model with benefits for caregivers and persons with dementia. It may lead to delay in institutionalization and may be applicable to other chronic conditions.

Key words: dementia, caregiver burden, psycho-social intervention, family caregiver, respite

\section{Introduction}

In 2017, approximately 413,000 people in Australia had a diagnosis of dementia, a number expected to rise to around 1.1 million by 2056 by which time current annual costs exceeding $\$ \mathrm{~A} 14$ billion annually are set to exceed $\$ \mathrm{~A} 1$ trillion (Brown et al., 2017); (1AUD $\approx 0.75 \mathrm{USD}$ ). It is estimated that dementia is the second leading cause of burden of disease and disability burden in Australia for people over the age of 65 years (AIHW, $2012 \mathrm{a}$ ). About $70 \%$ of people with dementia live in the community and receive informal care from family members or friends (AIHW, 2012a). Despite this, over half of directly attributable spending on dementia by the Australian Government was directed to residential aged care (AIHW, 2012b).

Correspondence should be addressed to: Ms Meredith Gresham, The Dementia Centre, Hammond Care, 97-115 River Road Greenwich, Sydney, New South Wales 2065, Australia. Phone: +61284377300. Email: mgresham@hammond.com.au. Received 17 Apr 2017; revision requested 5 Jul 2017; revised version received 10 Mar 2018; accepted 29 Mar 2018. First published online 18 July 2018.
This disparity in funding likely reflects the higher needs of people in long-term care as well as the informal contributions made by family caregivers.

The presence and ability of a caregiver to supervise and assist with core and non-core activities of daily living are critical factors in the ability of a person with dementia to remain at home (AIHW, 2012a). A meta-analysis of risk factors for admission to a residential aged care home (RACH) found older age and higher dementia severity increased risk, while living with a caregiver decreased risk (Cepoiu-Martin et al., 2016). Behavioral and psychological symptoms of dementia (BPSD) are also associated with the increased rate of placement in residential care and higher service utilization rates for caregivers (Rabinowitz et al., 2007; Schubert et al., 2008). Levels of caregiver stress have been shown to mediate the relationship of behavioral characteristics of the person with dementia and nursing home admission (Gaugler et al., 2011). This US study of over 5,000 caregivers highlighted the need for comprehensive intervention with both caregivers and the person 
with dementia to reduce the risk of nursing home admission for people with dementia.

Given the impact of burden on both caregivers and persons with dementia, a number of interventions have been designed to reduce burden through the provision of education for caregivers. Studies targeting this area have demonstrated effective impacts on caregiver depression, well-being, quality of life, and anxiety (Elvish et al., 2013). A randomized controlled trial of a structured tenday residential training program for caregivers and the person with dementia in Sydney, Australia demonstrated reduction in psychological distress of caregivers and delayed time to admission to residential care; $65 \%$ of people with dementia in the intervention group remained at home at 12month follow up compared to $26 \%$ of controls. In addition, the program demonstrated savings of an average of $\$ A 8,000$ per dyad (1991 figures) over the three years after training (Brodaty and Gresham, 1989; Brodaty and Peters, 1991). At seven-year follow up, effects of delaying admission to residential care and mortality were still evident (Brodaty et al., 1993).

Respite care, including residential respite, is considered a critical service to maintain people living with dementia at home (Bruen and Howe, 2009; AIHW, 2010a). Residential respite has been demonstrated to provide temporary relief from the burden of care (Neville and Byrne, 2008). However, the uptake of residential respite has remained low over time (Brodaty and Gresham, 1992; AIHW, 2010a) and residential respite is frequently a rehearsal for permanent entry to residential care with over $50 \%$ of all Australian residential respite stays converting to permanent placements within one year (AIHW, 2012b). A report commissioned by a major consumer advocacy organization, Alzheimer's Australia, (now Dementia Australia) investigated reasons for the low use of respite and concluded the lack of knowledge about available respite services, caregivers delaying use of respite and lack of flexibility of respite services inhibited their use and subsequent usefulness (Bruen and Howe, 2009).

Based on these findings, we designed a new program combining principles of the Sydney training program and respite care in nursing homes. The original program materials were updated in the light of advances in knowledge about dementia and new service arrangements.

This study reports an evaluation of the program, titled Going to Stay at Home. We hypothesized that after caregiver training

1. (conservatively, based on findings from our previous study - see above), that at least $60 \%$ of caregivers will have the person with dementia still living at home at 12-month follow-up,

2. caregiver psychological distress will be reduced at 6- and 12-months compared to baseline,

3. caregiver burden will be reduced at 6- and 12months compared to baseline, and

4. there will be no increase in behavioral or psychological symptoms of dementia in the person with dementia.

\section{Methods}

\section{Design}

Detailed methods have been previously reported (Gresham et al., 2014). Briefly, the Going to Stay At Home (GTSAH) evaluation is a single arm, preand post-study of a residential education program for caregivers of people with dementia. Outcome data were collected at three time points: at baseline and at 6- and 12-months after completion of the program.

\section{Setting}

A series of GTSAH programs ran for five days each for four to six dyads of persons with dementia and their primary family caregivers. Participants were admitted on a Sunday afternoon and stayed until the following Saturday morning. There were parallel sessions for caregivers and persons with dementia. GTSAH was conducted in an eight-bedroom, self-contained cottage, one of a cluster of eight secure cottages comprising a 91-place, Australian Government approved RACH in Sydney, Australia. Each dyad had their own motel style bedroom and ensuite bathroom. Living and dining facilities, kitchen, laundry, and garden were shared. Both caregiver and the person with dementia had all meals in the cottage. Social activities (e.g. classic movies, table tennis, music, and dancing) were arranged in the evening. The caregiver training program was conducted in an on-site staff training room. The people with dementia had a respite program focused on daily domestic, creative, and social activities in the cottage and outings. During the day and evening, the cottage was staffed by two nursing aides, who provided meals, snacks, social program support, and housekeeping. The cottage was monitored by an individually programmable infrared movement detection system at night, linked to a pager worn by the RACH night duty registered nurse $(\mathrm{RN})$. The $\mathrm{RN}$ would respond to movement outside the programmed parameters or activation of the nurse call system. To explore the "portability" of the GTSAH paradigm, one program was conducted in 
commercial serviced apartments in a regional town in New South Wales.

\section{The Going to Stay at Home (GTSAH) program}

The program for caregivers consisted of didactic sessions, small-group discussion, modeling of skills, and role play. Caregivers attended 14 sessions in total, focusing on psychological support to reduce distress, depression, and psychological morbidity; education and information about dementia and its progression; improving coping and home care skills, problem solving and behavioral management techniques; developing personal, family, community, and professional support systems; and planning for the future, including coping with emergencies, legal, and financial planning (Gresham et al., 2014).

The program for the person with dementia consisted of activities that focused on sensory and cognitive stimulation, physical activity, reminiscence, environmental orientation, creativity, social interaction, and relaxation. The cottage provided resources for domestic activity (e.g. kitchen, barbeque area, laundry, and garden) and outings were arranged in the local area with destinations based on personal interests of the participants. If participants were willing, one or more sessions were provided to discuss memory loss and the changes this had brought to participants' lives and relationships. Depending on need and willingness to receive information, individual or group interventions to help cope with memory loss were practiced such as using diaries and reminder systems, practicing simple mnemonic techniques, and utilizing environmental cues to provide prompts.

\section{Participants}

Participants of GTSAH were recruited through a broad promotional strategy that included referrals from the New South Wales Dementia Behaviour Management Advisory Services (now Dementia Support Australia), aged care community service providers, aged care assessment teams (ACAT) (regionally based teams of health professionals appointed to assess eligibility for Australian Government subsidised community and residential aged care services), health services, the media, and word of mouth. All participants with dementia were assessed by ACATs and deemed eligible for Australian Government funded residential respite care. After acceptance into the program, participants were placed into groups of 4-6 dyads. Where possible, groups were arranged by area of usual residence to facilitate support networks after the program (if desired), and by the age of the person with dementia.

After successful enrolment in the GTSAH program, dyads were invited to participate in the evaluation study. Participation in the evaluation was voluntary and participants could attend the program without agreeing to be part of the evaluation. For the evaluation study, inclusion criteria for the person with dementia were a diagnosis of dementia confirmed by their GP, specialist, or ACAT, residing at home with a primary caregiver (co-habiting or not), ability to communicate in English (at a basic level), and provision of either informed consent, or verbal assent plus proxy consent. Inclusion criteria for caregivers were the ability to communicate in English and the provision of informed consent.

\section{Staff}

Multi-skilled staff employed by the host RACH provided general support for meals, housework, laundry, and social activities in accordance with the host organization's model of care. A RACH RN was available as required for specific nursing advice or intervention. Medical support (if needed) was provided by a local general practice.

Program staff included a coordinator (physiotherapist) and assistant coordinator (activities officer) who were responsible for recruitment, some caregiver education sessions, and overall day-today operation of the program. Additional specialist sessional staff, including occupational therapists, a counselor, $\mathrm{RN}$, a psychologist, a dietician, and on occasion, a psychogeriatrician provided specific caregiver education content.

\section{Outcomes}

Information was collected through self-report and proxy questionnaires, as well as interviews with the caregiver. The primary outcomes were caregiver anxiety and depression measured using the modified ten-item Kessler Psychological Distress Scale (K-10) (Kessler et al., 2002).

Secondary outcomes were residential care placement at 12 months; caregiver burden (Zarit Burden Interview, short version) (Zarit et al., 1980; Bédard et al., 2001); caregiver quality of life (Older People's Quality of Life Questionnaire - Brief [OPQoL-Brief]) (Bowling et al., 2013); caregiver health status (Short Form Health Survey, SF12) (Ware et al., 1996); the number of caregiver needs met from an 11-item self-report needs scale (Harrison et al., 2014), and resource utilization (Resource Utilization in Dementia Lite [RUDLite]) (Wimo and Winblad, 2003). Outcomes to 
Table 1. Characteristics of the person with dementia (PWD) and caregiver

\begin{tabular}{|c|c|c|}
\hline & CHARACTERISTIC & VALUE \\
\hline Person with dementia $(n=90)$ & Age in years $(M, \mathrm{SD})$ & 75.4 (9.1) Range 49-90 \\
\hline \multirow{5}{*}{ Dementia diagnosis } & $\begin{array}{l}\text { Women }(n, \%) \\
\text { Alzheimer's disease }(n, \%)\end{array}$ & $\begin{array}{l}26(28.9) \\
52(58)\end{array}$ \\
\hline & Frontotemporal $(n, \%)$ & $11(12)$ \\
\hline & Lewy body $(n, \%)$ & $5(5)$ \\
\hline & Other $(n, \%)$ & $13(14)$ \\
\hline & Unknown $(n, \%)$ & $9(10)$ \\
\hline \multirow[t]{2}{*}{ Caregiver $(n=90)$} & Age in years $(M, \mathrm{SD})$ & 69.9 (10.8) Range 36-89 \\
\hline & Women $(n, \%)$ & $70(77.8)$ \\
\hline \multirow[t]{3}{*}{ Relationship to PWD } & Spouse & $78(86.7)$ \\
\hline & Child & $8(8.9)$ \\
\hline & Other & $4(4.4)$ \\
\hline
\end{tabular}

be measured related to the person with dementia were instrumental and basic activities of daily living (informant rated IADL and Physical Self Maintenance Scales) (Lawton and Brody, 1969); quality of life (proxy rated Quality Of Life - AD) (Logsdon et al., 1999; 2002); neuropsychiatric symptoms (Neuropsychiatric Inventory [NPI]) (Cummings et al., 1994); agitation (CohenMansfield Agitation Inventory [CMAI]) (CohenMansfield et al., 1995); and severity of dementia (Global Deterioration Scale [GDS]) (Reisberg et al., 1982). Global cognitive function of people with dementia was assessed at baseline by GTSAH staff using the Mini-Mental State Examination (MMSE) (Folstein et al., 1975).

\section{Rate of nursing home admissions in GTSAH participants and a comparison group}

Twelve-month rate of permanent admission to RACH amongst GTSAH participants was compared to the rate of permanent admission observed for people with a diagnosis of dementia who had been deemed by the ACAT as eligible and had a residential respite care stay in the same host organization, during the same time period as the GTSAH program. Comparison group admission rate to permanent residential care after respite was determined by an audit of the host organization's admission records.

\section{Statistical analysis}

The difference in outcome measures before and after intervention was examined using a oneway repeated ANOVA at an adjusted significance 0.0036 (two-sided) (Bonferroni adjustment for $\alpha 0.05,14$ comparisons). Logistic regression was used to compare rates of residential care admissions between program participants and a similar group that did not receive the program. Where applicable, missing items were handled as suggested in the literature for that particular instrument.

\section{Ethics}

The study was approved by the Human Research Ethics Committee, University of New South Wales, Sydney Australia (Approval number HC13033).

\section{Results}

\section{Program and participants}

The GTSAH program was offered to 131 dyads, with 90 entering the program and 41 applying but withdrawing before entering a program. All those who withdrew were offered a program at a later date. These participants are not included in the evaluation study as no consent was obtained for evaluation. In total, 19 GTSAH programs for groups of 3-6 dyads were completed between April 2013 and March 2014. Of the 90 dyads who completed baseline assessments, 85 completed 6month follow-up and 84 completed the 12-month follow-up. Four people with dementia had died by six months' follow-up and a fifth person with dementia died by 12 months. One dyad relocated internationally.

Characteristics for persons with dementia and caregivers are shown in Table 1. Of the ninety dyads, $75 \%$ came from the greater Sydney metropolitan region, $14 \%$ from areas outside of Sydney, and $11 \%$ remote or regional areas. The most common type of dementia was Alzheimer's disease and almost a quarter of participants with dementia $(22 \%)$ had younger onset dementia. 
Table 2. Caregiver and PWD outcomes by time

\begin{tabular}{|c|c|c|c|}
\hline & $\begin{array}{l}\text { BASELINE } \\
(N=90)\end{array}$ & $\begin{array}{l}6 \text { MONTH } \\
(N=85)\end{array}$ & $\begin{array}{l}12 \text { MONTH } \\
(N=84)\end{array}$ \\
\hline \multicolumn{4}{|l|}{ Caregiver } \\
\hline Caregiver K-10 (higher = worse) & $17.2(6.2)$ & $17.3(5.3)$ & $17.1(5.9)$ \\
\hline Caregiver Quality of life (higher = better) & $51.7(5.9)$ & $50.2(6.3)$ & $49.8(6.4)^{*}$ \\
\hline Caregiver burden (Zarit) (higher $=$ worse) & $19.6(8.1)$ & $20.2(8.0)$ & $20.1(8.6)$ \\
\hline SF-12 Physical Composite Score (PCS) & $46.6(11.3)$ & $45.2(11.5)$ & $45.9(11.6)$ \\
\hline SF-12 Mental Health Composite Score & $46.4(10.3)$ & $44.5(10.0)$ & $44.9(10.2)$ \\
\hline Rudlite hours (IADLs) & $5.3(6.3)$ & $6.1(6.3)$ & $5.9(6.4)$ \\
\hline Met needs, total score (higher $=$ met) & $5.6(2.6)$ & $7.6(2.4)$ & $8.2(1.9)^{*}$ \\
\hline \multicolumn{4}{|l|}{ Person with dementia } \\
\hline GDS $($ lower $=$ better $)$ & $4.4(0.8)$ & $4.8(0.9)$ & $4.9(0.9)$ \\
\hline PWD Quality of Life (higher = better) & $30.6(5.6)$ & $28.3(5.4)$ & $27.9(5.9)^{*}$ \\
\hline CMAI (lower $=$ better $)$ & $56.3(20.2)$ & $51.9(11.3)$ & $53.0(9.2)$ \\
\hline NPI total score (lower $=$ better) & $26.9(16.8)$ & $17.8(13.8)$ & $19.3(13.4)^{*}$ \\
\hline NPI number of symptoms $(\max 12)$ & $5.8(2.2)$ & $5.0(2.2)$ & $4.7(2.2)^{*}$ \\
\hline Lawton - IADLS (higher = better) & $2.8(1.5)$ & $2.1(1.6)$ & $1.6(1.5)^{*}$ \\
\hline Lawton - physical self-maintenance & $3.9(2.2)$ & $3.1(2.4)$ & $2.2(2.1)^{*}$ \\
\hline
\end{tabular}

${ }^{*} p<0.0036$ ( $\alpha 0.05$ Bonferroni adjustment for multiple comparisons).

Notes: SF-12, Short Form Health Survey; RUD-Lite, Resource Utilization in Dementia Lite; GDS, Global Deterioration Scale; NPI, Neuropsychiatric Inventory; CMAI, Cohen-Mansfield Agitation Inventory; GDS, Global Deterioration Scale; IADLS, Instrumental Activities of Daily Living Scale.

\section{Caregiver and person with dementia outcomes}

Outcomes for caregivers and person with dementia are shown in Table 2. There was no significant change in caregiver psychological distress (K-10), caregiver quality of life, caregiver burden (Zarit burden scale), SF-12 Physical composite score, or SF-12 Mental Health composite score over time.

For the person with dementia, there was an increase in Global Deterioration Scale scores (Greenhouse-Geisser correction $[\mathrm{F}(1.7,135.1)=$ 43.5, $p<0.001]$ ), and decrease in proxy rated person with dementia quality of life (GreenhouseGeisser correction $[\mathrm{F}(1.9,151.6)=18.5, p<$ $0.001]$, Lawton IADLs $[\mathrm{F}(2,156)=38.1 p<$ $0.001]$, and Lawton physical self-maintenance $[\mathrm{F}(2,156)=41.7 p<0.001])$. Post hoc tests (Bonferroni corrected) demonstrated deterioration from baseline at both 6- and 12-months. Across the same time period, however, there was a decrease in total NPI score $(\mathrm{F}(2,156)=16.9, p<0.001))$ and NPI number of symptoms $(\mathrm{F}(2,146)=6.4$, $p<0.001)$ ). Post hoc tests (Bonferroni corrected) showed both NPI scores were lower at 6- and 12months compared to baseline scores.

\section{Needs of caregiver}

As reported by caregivers, there was an increase in the number of their needs being met (GreenhouseGeisser correction, $\mathrm{F}(1.7,123.6)=26.9, p<$ $0.001)$. Post-hoc tests using a Bonferroni correction showed that at 6- and 12- months, met needs were higher than baseline. Specific needs (Table 3) relating to information, practical tasks, communication, and managing behavior were addressed but those concerning time and burden remained stable reflecting increasing functional dependence of the person with dementia.

\section{Impact on permanent admission to an aged care home}

At twelve months, $82.4 \%$ (70/85) of participants with dementia were still living in the community. This admission rate was compared to the rate for people with dementia receiving usual residential respite care in the same host organization during the same time period (Table 4). Of those with twelve-month data available, $52.9 \%$ had entered permanent care, compared to $17.6 \%$ of GTSAH participants. Only limited demographic and no clinical data were available for the comparison group.

To allow for people with dementia who received respite care in the comparison group but did not have follow-up data, we conducted a sensitivity analysis by generating a "conservative comparison" group. The conservative comparison group assumed that all people with dementia who received respite care but had missing data had remained living at home. This resulted in an estimated rate of permanent care of $44 \%$ within 12 months of usual respite care. A logistic regression model controlling for age and sex of the person with dementia was used to assess this association. 
Table 3. Change in caregiver self-reported met needs by item, over time

\begin{tabular}{|c|c|c|c|}
\hline NEED & $\begin{array}{l}\text { BASELINE } \\
\operatorname{MET}(N, \%) \\
N=78\end{array}$ & $\begin{array}{l}6 \text { MONTHS } \\
\text { MET }(\%) \\
N=74\end{array}$ & $\begin{array}{l}12 \text { MONTHS } \\
\text { MET }(\%) \\
N=75\end{array}$ \\
\hline Enough information about home services? & $54(73.0)$ & $59(81.9)$ & $63(86.3)$ \\
\hline $\begin{array}{l}\text { Knowledge of how to look after person in your care given } \\
\text { their diagnosis? }\end{array}$ & $31(40.8)$ & $62(87.3)$ & $68(91.9)$ \\
\hline Financial/legal entitlements & $49(63.6)$ & $63(85.1)$ & $58(79.5)$ \\
\hline Communication/management daily activities & $44(56.4)$ & $60(81.1)$ & $66(88.0)$ \\
\hline $\begin{array}{l}\text { Information and instructions on how to carry out practical } \\
\text { tasks for PWD }\end{array}$ & $43(59.7)$ & $66(94.3)$ & $71(97.3)$ \\
\hline Enough time for chores, other obligations? & $45(58.4)$ & $44(59.5)$ & $43(58.9)$ \\
\hline Time for yourself? & $26(33.8)$ & $31(42.5)$ & $37(50.7)$ \\
\hline Resources to manage critical events? & $38(79.2)$ & $45(86.5)$ & $45(90)$ \\
\hline Maintain existing networks & $39(50.6)$ & $42(57.5)$ & $40(54.1)$ \\
\hline Do you feel under increased emotional strain/burden? & $25(32.1)$ & $27(39.7)$ & $28(38.4)$ \\
\hline Adequate information on managing challenging behaviors? & $18(23.1)$ & $51(68.9)$ & $62(83.8)$ \\
\hline
\end{tabular}

Note. "Resources to manage critical events" had a high number of missing data across all times (approximately 30-40\%) possibly indicating caregivers either did not want to answer or had no experience with this.

Table 4. Comparison of residential aged care home (RACH) admissions over 12 months (GTSAH vs. usual respite)

\begin{tabular}{|c|c|c|c|c|c|}
\hline & $\begin{array}{l}\text { NOT IN } \\
\text { RACH AT } \\
12 \text { MONTHS }\end{array}$ & $\begin{array}{l}\text { IN RACH AT } \\
12 \text { MONTHS }\end{array}$ & $\chi^{2}$ & DF & $P$ \\
\hline GTSAH $^{1}$ & 70 & $15(17.6 \%)$ & 26.2 & 1 & $<.001$ \\
\hline Comparison group & 56 & $63(52.9 \%)$ & & & \\
\hline GTSAH & 70 & $15(17.6 \%)$ & 16.8 & 1 & $<.001$ \\
\hline Conservative comparison group ${ }^{2}$ & 79 & $63(44 \%)$ & & & \\
\hline
\end{tabular}

${ }^{1}$ GTSAH group does not include a participant who could not be contacted $(n=1)$ and those who were deceased $(n=4)$.

${ }^{2}$ Conservative comparison group assumes all unknown data in the comparison group have remained out of permanent care at 12 months.

The outcome was being admitted to an $\mathrm{RACH}$ compared to not being admitted to an RACH in 12 months. Those in the conservative comparison group were more likely to be in a residential care home at twelve months (OR 5.8, 95\% CI 2.8-11.6, $p<0.001)$.

\section{Discussion}

The Going to Stay at Home program aimed to provide a practical, intensive, and comprehensive residential caregiver training course to assist family caregivers develop and hone their skills to enable people with dementia to successfully remain at home. The program was modeled on the successful Sydney dementia caregivers training program (Brodaty and Gresham, 1989; Brodaty et al., 1997) but was conducted as an adjunct to a residential respite stay in an Australian Government funded RACH. We confirmed two of our hypotheses that: more than $60 \%$ of persons with dementia would still be living at home at 12-month follow-up, and that there would be no increase in BPSD. However, contrary to two of our hypotheses, there was no observed reduction in either caregiver psychological distress or caregiver burden over follow-up. As expected, dementia severity increased as reflected in functional ability over the 12 months, but against this trend, behavioral symptoms improved.

Caring for a family member living with dementia is time consuming and frequently stressful and the reasons for admission of someone with dementia to long-term care are complex. We had hypothesized that if people with dementia were to remain living at home successfully, we would also observe reduced rates of caregiver burden and distress (known risk factors for RACH admission). While we did not demonstrate a reduction in caregivers' psychological distress of caregivers, there was also no increase over 12 months. Likewise, the burden associated with care did not increase despite the decrease of the abilities of the person living with dementia to carry out activities of daily living 
and physical self-maintenance. Over the evaluation period, caregivers reported a decrease in their quality of life (not significant after correction for multiple comparisons) and little change in time spent on chores and other obligations. There was a small increase in those who reported that they had enough time for themselves and maintenance of their existing social networks.

Although the program contained but did not reduce burden or distress, it was able to deliver effective education and information about dementia and its progression, improve caregivers' coping and home care skills, problem-solving and behavioral management techniques. Of note was the increase in meeting caregiver needs regarding knowledge and information about looking after a person with dementia, particularly concerning managing challenging behaviors.

The impact of the program on meeting needs around managing challenging behaviors may have contributed to our finding of a decrease in BPSD (consistent with our hypothesis) during follow-up even as dementia progressed. The effects of BPSD on caregivers can vary based on how unpredictable, uncontrollable, and distressing these are for the caregiver (e.g. Rabinowitz et al., 2007) and BPSD are a very common reason given by informal caregivers for the transition to permanent care (Afram et al., 2014). The success of the current program in meeting caregiver needs around BPSD may have been an important factor in ensuring caregiver burden did not increase and that people with dementia remained living at home. Further to this, self-efficacy amongst caregivers is associated with a reduced burden associated with frequent BPSD (Nogales-González et al., 2015). Although our evaluation did not include a direct measure of self-efficacy, increased knowledge can improve self-efficacy (Nogales-González et al., 2015) and meeting needs may have been a driver in the usefulness of the GTSAH program. Including a measure of self-efficacy in future studies could provide a direct test of this assertion.

The residential nature and inclusion of people with dementia in caregiver training are novel. Anecdotally caregivers reported that being in residence was beneficial, avoiding the stress of repeatedly getting prepared and travelling as well as having to find respite for the person with dementia while attending training. Caregivers appreciated being relieved of domestic tasks (e.g. cooking and cleaning). They commented that time with other caregivers and staff was valuable to decrease their sense of isolation; provide an opportunity to try new skills in a supportive environment; and to observe staff model skills. GTSAH did not have specific interventions for the person with dementia, but a program of pleasant activities tailored to the interests of each group. Caregivers reported greater engagement of the person with dementia with people and the environment after their return home.

Chronic disease, including dementia, has major, long-term impacts on the person who has the disease, their caregiver, and health and social systems. Current health policy in Australia largely focuses on the treatment of illness, rather than prevention or proactive management of chronic conditions, a scenario that has been described as a threat which may overwhelm health systems and budgets as the population ages (Willcox, 2014). While the incidence of dementia has been reported to be falling in several countries, the prevalence of dementia will continue to rise (Brown et al., 2017). Widespread adoption of multifaceted caregiver training programs, such as GTSAH, may provide a strategy to support caregivers to manage the person with chronic illness at home more effectively, as well as to manage their own health and well-being. This may reduce the use of health and community services. GTSAH has been adopted by the host organization as an ongoing service offering, on a user-pays basis or as part of a consumer directed package of community care package. GTSAH is costed at about AUD3000 (2017 figures) per dyad. Total systems cost for a person with dementia living in residential care has been estimated at AUD88,000 per annum or AUD241.00 per day (2016 figures) of which $94 \%$ of the costs were attributed to residential care (Gnanamanickam et al., 2018) In other words, staying out of a RACH for 13 days would over the cost of the program. Larger, controlled, longitudinal studies that also involve economic analyses are required.

\section{Limitations}

There are limitations to this study. First, this study was single arm due to funding requirements for the evaluation of program implementation that precluded a control group. While we cannot be certain of comparability of the comparison group, both groups were deemed eligible for residential respite by the ACAT.

Instead, a comparison group was used to provide two estimates (actual and conservative) of RACH admissions across the follow-up period. We acknowledge that differences in recruitment procedures and characteristics of persons with dementia may have influenced the rates of permanent placement and that the study sample had volunteered or agreed to be in the study and may not be representative of caregivers 
generally. Further, while we relied on an audit of facility records to determine the rates of admission to permanent care from respite stay in the comparison group, their rate of $53 \%$ is consistent with Australian data for people with dementia following respite use (55\%) (AIHW, $2010 \mathrm{~b}$ ). It is also likely that our reliance on audits from one service provider rather than direct followup underestimated rates of institutionalization in the comparison group makes our comparisons conservative. Additionally, our sensitivity analysis in which we assumed all the comparison persons with dementia who were not admitted to the host facility were still living in the community is a very conservative estimate against this standard (44\%). The GTSAH program performs well against our comparison and conservative comparison groups as well as reported population admission to care following respite (AIHW, 2010b).

A further limitation, as previously discussed, is that we were unable to conduct a cost benefits analysis.

\section{Strengths}

While the results largely apply to dyads in the greater Sydney metropolitan area (with one regional group), the study needs to be replicated in other areas and other cultural groups. The GTSAH program demonstrated in-principle portability through the successful conduct of a program in a regional town of NSW. We note the high number of people with younger onset dementia, probably because they or their families had better social media connections and may have been more aware of the program through this medium. In one case, an entire younger onset support group ( $\mathrm{n}=6$ dyads) elected to attend the program as a group.

The program demonstrated face validity. Of the 90 participating dyads who commenced the program, only one did not complete it. This caregiver was unable to participate in a meaningful way due to exacerbation of his chronic illness and was discharged with appropriate community and medical services in place. As previously reported, the only reason for non-completion of follow-up was the death of the person with dementia moving internationally.

GTSAH has provided a clear and workable model to help keep people with dementia living at home and to assist families to cope with this chronic and deteriorating condition. The GTSAH program provides a promising framework to enable family caregivers to keep their person with dementia at home longer. This was observed despite no reduction in caregiver burden and the expected deterioration over time in functional independence of persons with dementia. This proactive intervention model may have significant potential for application to people living with other chronic diseases.

\section{Conflict of interest}

Authors Gresham and Heffernan have no conflicts to declare. Professor Brodaty is an advisory board member for Nutricia.

\section{Description of authors' roles}

MG developed and managed the program and supervised data collection. $\mathrm{MH}$ and $\mathrm{HB}$ designed the evaluation and analyzed the data. All authors were involved in drafting and editing the paper.

\section{Acknowledgments}

We would like to acknowledge the significant contribution of the participants and staff of the GTSAH program, HammondCare for hosting the program and the Dementia Collaborative Research Centre- Assessment and Better Care at the University of NSW that conducted the evaluation. Special thanks to Deborah Moore. The project was funded by the Australian Government Department of Social Services under the Aged Care Services Improvement and Healthy Ageing Grants Flexible Fund, grant funding round DoHA/095/1112.

\section{References}

Afram, B. et al. (2014). Reasons for institutionalization of people with dementia: informal caregiver reports from 8 European countries. Fournal of the American Medical Directors Association, 15, 108-116. doi: 10.1016/j.jamda.2013.09.012.

AIHW (2010a). Dementia and the Take-up of Residential Respite Care. AIHW Bulletin No. 78. Cat. No. AUS 124. Canberra: Australian Institute of Health and Welfare.

AIHW (2010b). Residential Aged Care in Australia 2008-09: a Statistical Overview. Aged Care Statistics Series No. 31. Cat. no. $A G E$ 62. Canberra: Australian Institute of Health and Welfare.

AIHW (2012a). Dementia in Australia. Cat. No. AGE 70. Canberra: Australian Institute of Health and Welfare.

AIHW (2012b). Residential Aged Care in Australia 2010-11: a Statistical Overview. Age Care Statistics Series No. 36. Cat. No. $A G E$ 68. Canberra: Australian Institute of Health and Welfare.

Bédard, M., Molloy, D. W., Squire, L., Dubois, S., Lever, J. A. and O'Donnell, M. (2001). The Zarit burden interview: a new short version and screening 
version. Gerontologist, 41, 652-657. doi:

10.1093/geront/41.5.652.

Bowling, A., Hankins, M., Windle, G., Bilotta, C. and Grant, R. (2013). A short measure of quality of life in older age: the performance of the brief older people's quality of life questionnaire (OPQOL-brief). Archives of Gerontology and Geriatrics, 56, 181-187. doi:

10.1016/j.archger.2012.08.012.

Brodaty, H. and Gresham, M. (1989). Effect of a training programme to reduce stress in carers of patients with dementia. British Medical fournal, 299, 1375-1379.

Brodaty, H. and Gresham, M. (1992). Prescribing residential respite care for dementia-effects, sideeffects, indications and dosage. International fournal of Geriatric Psychiatry, 7, 357-362. doi: $10.1002 /$ gps.930070509.

Brodaty, H., Gresham, M. and Luscombe, G. (1997). The Prince Henry Hospital dementia caregiver's training program. International fournal of Geriatric Psychiatry, 12, 183-192. doi: 10.1002/(SICI)10991166(199702)12:2<183::AID-GPS584>3.0.CO;2-J.

Brodaty, H., McGilchrist, C., Harris, L. and Peters, K. E. (1993). Time until institutionalization and death in patients with dementia: role of caregiver training and risk factors. Archives of Neurology, 50, 643-650. doi: 10.1001/archneur.1993.00540060073021.

Brodaty, H. and Peters, K. E. (1991). Cost effectiveness of a training program for dementia carers. International Psychogeriatrics, 3, 11-22. doi: 10.1017/S1041610291000479.

Brown, L., Hansnata, E. and La, H. A. (2017). Economic Cost of Dementia in Australia 2016-2056. Canberra: National Centre for Social and Economic Modelling.

Bruen, W. and Howe, A. (2009). Respite Care for People living with Dementia. It's More Than fust a Short Break. Canberra: Alzheimers Australia.

Cepoiu-Martin, M., Tam-Tham, H., Patten, S., Maxwell, C. J. and Hogan, D. B. (2016). Predictors of long-term care placement in persons with dementia: a systematic review and meta-analysis. International fournal of Geriatric Psychiatry, 31, 1151-1171. doi: $10.1002 /$ gps.4449.

Cohen-Mansfield, J., Werner, P., Watson, V. and Pasis, S. (1995). Agitation among elderly persons at adult day-care centers: the experiences of relatives and staff members. International Psychogeriatrics, 7, 447-458. doi: 10.1017/s1041610295002195.

Cummings, J. L., Mega, M., Gray, K., Rosenberg-Thompson, S., Carusi, D. A. and Gornbein, J. (1994). The Neuropsychiatric Inventory: comprehensive assessment of psychopathology in dementia. Neurology, 44, 2308-2314. doi: 10.1212/wnl.44.12.2308.

Elvish, R., Lever, S.-J., Johnstone, J., Cawley, R. and Keady, J. (2013). Psychological interventions for carers of people with dementia: a systematic review of quantitative and qualitative evidence. Counselling and Psychotherapy Research, 13, 106-125. doi 10.1080/14733145.2012.739632.

Folstein, M. F., Folstein, S. E. and McHugh, P. R. (1975). "Mini-mental state": a practical method for grading the cognitive state of patients for the clinician. fournal of
Psychiatric Research, 12. doi: 10.1016/0022-3956(75)90026-6.

Gaugler, J. E., Kane, R. L., Kane, R. A., Clay, T. and Newcomer, R. (2011). Does caregiver burden mediate the effects of behavioral disturbances on nursing home admission? American fournal of Geriatric Psychiatry, 19, 497-506. doi: 10.1097/JGP.0b013e31820d92cc.

Gnanamanickam, E. S. et al. (2018). Direct health and residential care costs of people living with dementia in Australian residential aged care. International fournal of Geriatric Psychiatry. Epublished ahead of print, doi: $10.1002 /$ gps. 4842 .

Gresham, M., Tsang, R. S. M., Heffernan, M. and Brodaty, H. (2014). Study protocol of the going to stay at home program: evaluation of a residential carer training program to reduce dementia carer distress and burden. SpringerPlus, 3, 1-7. doi: 10.1186/2193-1801-3-330.

Harrison, F., Low, L.-F., Barnett, A., Gresham, M. and Brodaty, H. (2014). What do clients expect of community care and what are their needs? The community care for the elderly: needs and service use study (CENSUS). Australasian fournal on Ageing, 33, 208-213. doi: 10.1111/ajag.12118.

Kessler, R. C. et al. (2002). Short screening scales to monitor population prevalences and trends in non-specific psychological distress. Psychological Medicine, 32, 959-976. doi: $10.1017 /$ S0033291702006074.

Lawton, M. P. and Brody, E. M. (1969). Assessment of older people: self-maintaining and instrumental activities of daily living. Nursing Research, 19, 179-186.

Logsdon, R. G., Gibbons, L. E., McCurry, S. M. and Teri, L. (1999). Quality of life in Alzheimer's disease: patient and caregiver reports. Fournal of Mental Health and Aging, 5, 21-32.

Logsdon, R. G., Gibbons, L. E., McCurry, S. M. and Teri, L. (2002). Assessing quality of life in older adults with cognitive impairment. Psychosomatic Medicine, 64, 510-519. doi: 10.1097/00006842-200205000-00016.

Neville, C. C. and Byrne, G. J. A. (2008). Effect of a residential respite admission for older people on regional Queensland family carers. Collegian, 15, 159-164. doi: 10.1016/j.colegn.2008.06.003.

Nogales-González, C., Romero-Moreno, R., Losada, A., Márquez-González, M. and Zarit, S. H. (2015).

Moderating effect of self-efficacy on the relation between behavior problems in persons with dementia and the distress they cause in caregivers. Aging and Mental Health, 19, 1022-1030. doi: 10.1080/13607863.2014.995593.

Rabinowitz, Y. G., Mausbach, B. T., Thompson, L. W. and Gallagher-Thompson, D. (2007). The relationship between self-efficacy and cumulative health risk associated with health behavior patterns in female caregivers of elderly relatives with Alzheimer's dementia. fournal of Aging and Health, 19, 946-964. doi: 10.1177/0898264307308559.

Reisberg, B., Ferris, S. H., de Leon, M. J. and Crook, T. (1982). The global deterioration scale for assessment of primary degenerative dementia. American fournal of Geriatric Psychiatry, 139, 1136-1139. doi: 10.1176/ajp.139.9.1136. 
Schubert, C. C., Boustani, M., Callahan, C. M., Perkins, A. J., Hui, S. and Hendrie, H. C. (2008). Acute care utilization by dementia caregivers within urban primary care practices. Fournal of General Internal Medicine, 23, 1736-1740. doi: 10.1007/s11606-008-0711-0.

Ware, J. E., Kosinski, M. and Keller, S. D. (1996). A 12-Item short-form health survey: construction of scales and preliminary tests of reliability and validity. Medical Care, 34, 220-233. doi:

10.1097/00005650-199603000-00003.
Willcox, S. (2014). Chronic diseases in Australia: the case for changing course. Australian Health Policy Collaboration Issues Paper No. 2014-02. Melbourne: Australian Health Policy Collaboration.

Wimo, A. and Winblad, B. (2003). Resource utilization in dementia: RUD Lite. Brain Aging, 3, 48-59.

Zarit, S. H., Reever, K. E. and Bach-Peterson, J. (1980). Relatives of the impaired elderly: correlates of feelings of burden. The Gerontologist, 20, 649-655. doi: 10.1093/geront/20.6.649. 